\title{
Anabases
}

ANABASES Traditions et réceptions de l'Antiquité

$21 \mid 2015$

Varia

\section{Almut-Barbara RENGER et Jon SOLOMON, Ancient Worlds in Film and Television: Gender and Politics}

Mathieu Scapin et Matthieu Soler

\section{(2)enEdition}

\section{Journals}

Édition électronique

URL : http://journals.openedition.org/anabases/5363

DOI : $10.4000 /$ anabases. 5363

ISSN : 2256-9421

\section{Éditeur}

E.R.A.S.M.E.

\section{Édition imprimée}

Date de publication : 1 avril 2015

Pagination : 285-287

ISSN : 1774-4296

\section{Référence électronique}

Mathieu Scapin et Matthieu Soler, « Almut-Barbara renger et Jon Solomon, Ancient Worlds in Film and Television: Gender and Politics », Anabases [En ligne], 21 | 2015, mis en ligne le 01 avril 2015, consulté le 22 septembre 2020. URL : http://journals.openedition.org/anabases/5363 ; DOI : https://doi.org/ $10.4000 /$ anabases.5363

Ce document a été généré automatiquement le 22 septembre 2020

(c) Anabases 


\title{
Almut-Barbara RENGER et Jon SOLOMON, Ancient Worlds in Film and Television: Gender and Politics
}

\author{
Mathieu Scapin et Matthieu Soler
}

\section{RÉFÉRENCE}

Almut-Barbara RENGER et Jon SOLOMON, Ancient Worlds in Film and Television: Gender and Politics, Leiden-Boston, Brill, Metaforms, 2013, 332 p.

123 euros / ISBN 9789004183209

1 Le premier Metaforms est centré sur le sens sociopolitique d'un cinéma à l'Antique mettant en scène des corps/images reflets des relations de genre. Ce livre résulte d'un colloque international pluridisciplinaire tenu à la Freie Universität Berlin en décembre 2009. Seize interventions sont publiées, étudiant ces recompositions en prenant en compte conditions et codes de production, stratégies de vente, autocensures et pressions socioculturelles. L'ouvrage est scindé en trois parties déséquilibrées et peu cohérentes, oubliant parfois la relation politique/genre. Un découpage thématique appuyé sur les développements majeurs des différents articles aurait été pertinent : corps-icône, instrumentalisation politique, manipulation des codes.

Historical Ancients parle de films fondés sur des événements historiques, éludant la question des sources - renvoyée à M. Winkler. Dans « Ben-Hur and Gladiator : Manifest destiny and the contradictions of American empire ", J. Solomon, à travers deux œuvres majeures, pose la méthodologie et montre la complexité et la multivalence de la relation entre les messages $\mathrm{du}$ film et son audience, permettant à un public fragmentaire de choisir ce qu'il veut voir et entendre. L'attraction-répulsion des Américains envers l'impérialisme s'y lit dans l'archétype du personnage, infléchi par la personnalité et les engagements de l'acteur (Th. Späh et M. Tröhler, « Muscles and morals : Spartacus, ancient hero of modern times »). Cette centralité de l'image du 
corps se retrouve dans le controversé 300 de Zack Snyder. L'héroïsme mâle de Léonidas, par ailleurs père et mari, ne s'accomplit qu'à travers sa femme, Gorgo, forte et moderne (T. Beigen, « With your shield or on it : The gender of heroism in Zack Snyder's 300 and Rudolph Maté's The 300 Spartans »). Il y a là une véritable interrogation sur la domination du discours masculin dans notre société (M. Dhont, J. Lauwers et X. Huybrecht, « This is Sparta! : Discourse, gender, and the Orient in Zach Snyder's 300 »). La narration de Snyder met en avant la bataille idéologique, livrant la vision des faits telle que les Spartiates auraient pu la conter. Il faut donc passer outre le racisme, l'homophobie, ou la vulgarité (excellent R. J. Poole, « Everybody loves a muscle boy »). Ce même type de déconstruction du rapport féminin/masculin se retrouve dans Rome (M. M. Toscano, "The womanizing of Mark Antony: virile ruthlessness and redemptive cross-dressing in Rome, Season Two »). Marc Antoine, symbole de la force mâle, s'oppose à la douceur et à la vulnérabilité méprisées et féminisées. Les femmes de son entourage le changent jusqu'au travestissement final qui le place au-dessus et dans les catégories d'opposés. Il y gagne sa rédemption tandis qu'Octave, masculinisé, perd son âme : à quel prix est le pouvoir ? C'est là une inversion du point de vue qui prévaut dans les réceptions modernes : image d'un Orient lascif, vision moderne de l'Est (E. Bronfen, « Cleopatra's Venus »).

3 Dans Mythological Ancients, la mythologie est utilisée pour interroger la modernité des relations masculines et des frontières littéraires et cinématographiques qui les régissent dans Troie (A. Kraas dans « Over his dead body : male friendship in Homer's Iliad and Wolfgagng Petersen's Troy (2004)»). L'amitié passionnée de l'Iliade, Patrocle/ Achille, est changée en lien familial obscur dans le film, laissant le spectateur choisir le message (C. Proche et M. Kleu, "Models of masculinities in Troy »). Malgré cette richesse sémantique, le poème épique d'Homère reste un récit marginal, adapté par Camerini, Godard et Rossi (C. Pischel, "Include me out ») qui construisent des conceptions de l'épique, transformant expérience théâtrale ou littéraire en discours cinématographique. Pasolini fait de même, sa Médée, par sa masculinité héroïque, est un anti-modèle utilisé pour dénoncer l'anachronisme du rejet des transgressions en 1969 (L. Stavanicic, "Between mythical and rational worlds »). Celui-ci est visible lors de la protestation de croyants hindous contre la mise en scène d'une relation homosexuelle recevant l'assentiment de Krishna dans Xena (X. Zeiler, "Universal's religious bigotry against hinduism »).

Mythological and Historical Thematics invite à s'extraire du péplum pour explorer des films qui auraient un fond de tradition classique. S. SlapŠak propose une analyse fondée sur l'anthropologie historique française (" Ancient woman's cults and rituals in grand narratives on screen from Walt Disney's snow white to Olga Malea's Doughnuts with honey »). La posture de l'auteur étonne quand elle compare, sans convaincre, l'iconographie grecque à Blanche Neige, et élude la question de la culture classique des auteurs. Il nous semble impossible, méthodologiquement, de faire l'économie d'une réflexion sur Andersen et ses réceptions, avant de renvoyer à une Antiquité intemporelle qui serait indissociable de notre inconscient. Les articles suivants, de meilleure tenue, présentent des femmes-icônes. Avec Pandora, Hésiode devient le prétexte à une mise en scène du regard du réalisateur sur Ava Gardner (A.-B. Renger, " Pandora-Eve-Ava : Albert Lewin's Making of a "Secret Goddess" »). Actrice et personnage deviennent une figure christique de la rédemption et du sacrifice (B. Schrödl, "Phryne paves the way for the wirtschaftswunder : vision of guilt and "purity" fed by the Ancient Greece, Christian narrative, and contemporary history » et T. Ilan « 
The new israeli film Beruriah: between Rashi and Talmud, between Antiquity and Modernity, between feminism and religion »).

5 L'ouvrage est doté d'un index et de photographies couleur petites et simplement illustratives. Le lecteur averti aimerait trouver dans le livre les spécialités des auteurs afin de mieux cerner leur regard, de comprendre comment telle spécialité travaille et envisage ses méthodes. Cet ouvrage n'est pas destiné à tous les publics, les chercheurs n'y trouveront que ponctuellement matière à réfléchir et les publics érudit ou étudiant s'égareront dans le manque global de cohérence.

\section{AUTEURS}

\section{MATHIEU SCAPIN}

Université de Toulouse - Jean Jaurès (UT2J)

mathieu.scapin@gmail.com

soler.matthieu@laposte.net 